Conclusions Biomarkers serum protein DKK1 and DKK1 expression have validity in diagnosis and post-treatment of HCC, especially for AFP-negative patient. HBx-LINE1 fusion transcript was not identified in our study.

\section{IDDF2019-ABS-0125 HOST GENETIC FACTORS ASSOCIATED WITH HEPATITIS C SPONTANEOUS VIRAL CLEARANCE: A META-ANALYSIS}

${ }^{1}$ Dewi Nur Aisyah*, ${ }^{2}$ Laura Shallcross, ${ }^{3}$ Alice Jane Hully, ${ }^{4}$ Alastair O'Brien, ${ }^{5}$ Andrew Hayward. ${ }^{1}$ Faculty of Public Health, Universitas Indonesia, Indonesia; ${ }^{2} \mathrm{UCL}$ Infectious Disease Informatics, Farr Institute of Health Informatics, UK; ${ }^{3}$ Kings College London School of Medicine, UK; ${ }^{4}$ UCL Division of Medicine, UK; ${ }^{5}$ Institute of Epidemiology and Health Care, University College London, UK

\subsection{6/gutjnl-2019-IDDFabstracts.91}

Background In the age of Direct Acting Antiviral (DAA) treatment where the cost of treatment is still very high, it may make sense to prioritise early treatment for individuals who are unlikely to achieve spontaneous clearance. Host genetic predictors might help us to select the right candidate, as several potential host genetic determinants have proven as the strongest indicators of clearance. Thus, genetic testing may help select individuals that would profit most from immediate treatment (high risk of progression and/or low risk of clearance), versus individuals who could be given a chance to see if spontaneous clearance occurs. The aim of this study is to synthesise available studies to provide a more precise estimate of the role of genetic factors in hepatitis spontaneous viral clearance (SVC).

Methods We performed a systematic search that reported for studies reporting the association between host genetic factors and SVC in 3 major databases (Ovid Medline, Ovid Embase, and Pubmed) between $1^{\text {st }}$ January 1994 and $30^{\text {th }}$ June 2015. We calculated odds ratios comparing patients who had achieved SVC to individuals who had not for each genetic predictor and undertook meta-analysis to identify genetic factors that were associated with clearance.

Results 86 studies met the inclusion criteria, representing 38,605 individuals. We identified 146 genetic factors, 24 of which were strongly associated with clearance. After we tabulate the odds ratio for SVC against allele frequency in those who cleared spontaneously, the strongest host genetic predictors of clearance were IL28B rs8103142 (OR=4.06, 95\% CI: 2.64-6.25), IL28B rs12979860 (OR=3.27, 95\% CI: 2.68-3.98), and IL28B rs8099917 (OR=2.83, 95\%CI: 2.363.39). (figure 1)

Conclusions This study suggests that patients with unfavorable IL28B genotypes could be given priority for early treatment with direct acting antivirals, given their low likelihood of spontaneous clearance. IL28B typing might help to inform the decision to start therapy in patients with chronic genotype 1 $\mathrm{HCV}$ infection, particularly in the context of the limited availability of DAA therapy in many places. However, the benefits of genotyping would need to be considered in the context of the falling costs of DAA's treatment.

\section{IDDF2019-ABS-0127 ASSESSING HCV DISTRIBUTION AMONG 'HARD TO REACH' POPULATIONS IN LONDON USING WHOLE GENOME SEQUENCING: REPORT FROM THE TB REACH STUDY}

${ }^{1}$ Dewi Nur Aisyah*, ${ }^{2}$ Laura Shallcross, ${ }^{3}$ Zisis Kozlakidis, ${ }^{2}$ Myrto Kremyda Vlachou ${ }^{4}$ Andrew Hayward. 'Faculty of Public Health, Universitas Indonesia, Indonesia; ${ }^{2}$ UCL Infectious Disease Informatics, Farr Institute of Health Informatics Research, UK; ${ }^{3}$ University College London, Division of Infection and Immunity, UK; ${ }^{4}$ Institute of Epidemiology and Health Care, University College London, UK

\subsection{6/gutjnl-2019-IDDFabstracts.92}

Background Hepatitis C virus (HCV) probably evolves and is transmitted in micro-epidemics within geographically or socially defined communities. Thus it is likely that the genomic information from HCV-positive participants, when combined to the extant epidemiological characteristics, might provide some insight into HCV transmission between high-risk individuals.

Methods Samples were collected from 39 homeless hostels, 20 drug treatment services and a prison between May 2011 and June 2013 in London, United Kingdom. Blood samples were collected from participants to be tested at the Royal Free Hospital for HCV. RNA extraction was performed from residual diagnostic specimens and processed locally within the hospital virology laboratories for PCR library preparation and Next Generation Sequencing using Illumina MiSeq equipment. The QIAamp Virus BioRobot MDx Kits were

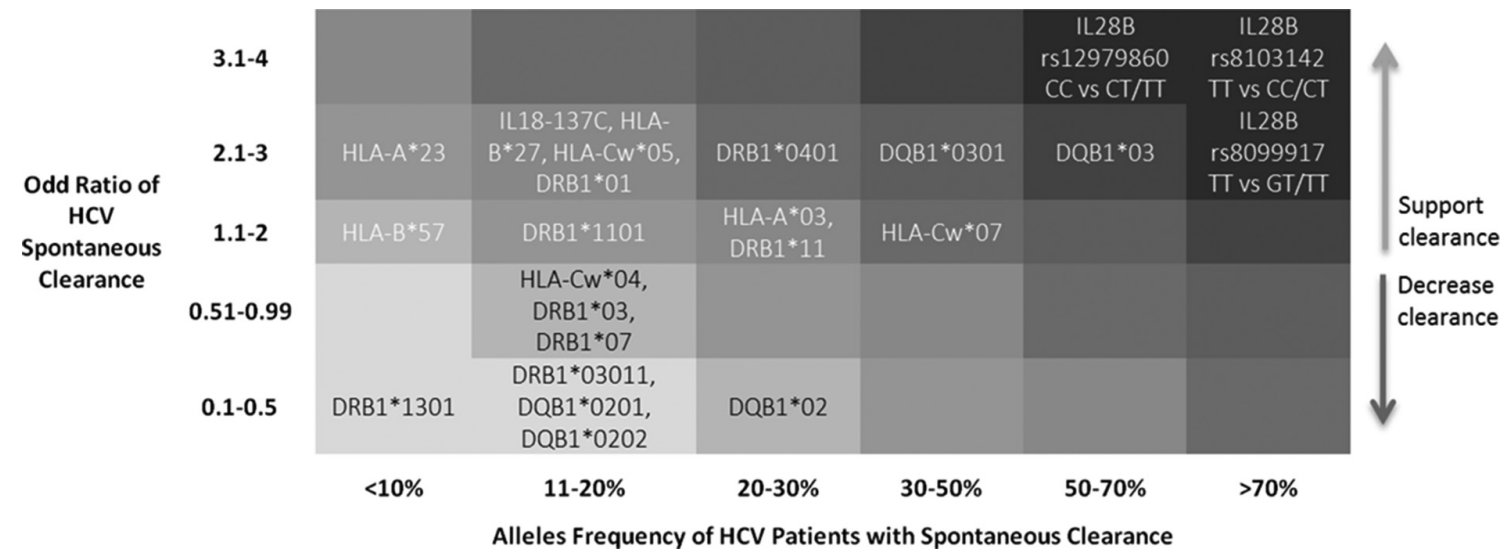

Abstract IDDF2019-ABS-0125 Figure 1 Odd ratio of hcv spontaneous clearance in relation to allele frequency 